\title{
TOWARDS 3D FACIAL RECONSTRUCTION USING DEEP NEURAL NETWORKS
}

\author{
Hafiz Muhammad Umair Munir and Waqar S. Qureshi \\ Department of Mechatronics, National University of Sciences and Technology, H-12 Islamabad
}

\begin{abstract}
3D facial reconstruction is an emerging and interesting application in the field of computer graphics and computer vision and is been used for 3D printing, creating avatars etc. It is difficult and challenging to reconstruct the 3D model of face from a single photo because of arbitrary poses, non-uniform illumination, expressions and occlusions. None of algorithm provides detailed 3D facial model because every algorithm has some limitations related to profile view, fine detail, accuracy and speed. The major problem is to develop 3D face with texture of large poses, wild faces and occluded faces. Mostly algorithm is used convolution neural network (CNN) and deep learning frameworks to create facial model and 3D dense face alignment (3DDFA) is the first algorithm that constructed the database consisting of 2D images and 3D facial model. In this paper we review 3D face reconstruction algorithm for application such as 3D printing, creating avatars and facial recognition. Different issues, problems and their proposed solutions are discussed in this paper while advantages and disadvantages are highlighted. A comparison of different algorithms is described in the context of texture and poses to find the best solution regarding reconstruction of 3D facial model from single photo.
\end{abstract}

\section{KEYWORDS}

3D Facial Model, Computer Vision, Convolution Neural Network, Deep Learning Frameworks

\section{INTRODUCTION}

Mostly algorithms for 3D facial shape is used only for small poses, medium poses, and uniform illumination but challenge comes when there is profile view and occluded view. Current algorithms and system are using multiple images as an input and getting more fine details as compared to using single image as an input. The computational cost of multi-view algorithm is always high. Constructing the 3D facial model from a single photo is still a problem and researchers are going to solve that problem. Existing algorithms of facial reconstruction are designed that are based on variety of models such as landmark-based face model and 3D Morphable Models (3DMM). This paper focuses on 3D facial reconstruction from single image using deep neural network architecture. Single photo face reconstruction is rarely employed by modern face recognition systems. 3DMM is the most popular method for reconstructing the full 3D facial model from a single photo. CNN has applications in the field of image classification, object detection and extract meaningful information from photos.

The earliest approaches is utilized the reference 3D faces (Hassner, Basri, 2006) (Hassner, Basri, 2013) to adjust the shape estimated for an input face image. These old methods are emphasized on robustness rather than fine details. Later methods are designed to construct the 3D face model by detection of facial landmarks (Jourabloo, Liu, 2016) (Zhu, 2016) and these algorithms give importance to accuracy regarding detection of landmarks. The 3DMM fitting methods are widely used which is originally proposed by Blanz and Vetter (Blanz, Vetter, 1999). Shape from shading techniques (Kemelmacher-Shlizerman, Basri, 2011) (Li, 2014) showed accurate and detailed 3D face reconstruction. Now a day deep neural networks (Jackson, 2017) (Tran, 2017) (Tran, 2018) are used for the estimation of 3D face shape with an unconstrained images. These deep 3D face estimation methods are still challenging and focus on speed, accuracy and low computational cost. Nikolai et al. (Nikolai, 2018) proposed mobile based 3D facial reconstruction which is fast. Coarse-to-fine method is used to reconstruct a high-quality 3D facial model from a single photo (Jiang, 2018) but whole face is reconstructed during reconstruction. Self-supervised bootstrap method (Xing, 2018) and 
model free approach (Feng, 2018) is new technique for 3D face reconstruction. The four algorithms with their features, pros and cons will be explained later.

There are four main challenges that are occurring now a day in 3D face reconstruction methods. Firstly, commonly landmark-based face model is used but this method is not suitable for profile views because appearance of face dramatically changes from frontal view to profile view. Secondly, in large poses landmark labeling is extremely challenging task that invisible landmarks have to be guessed. Thirdly, 3D estimates of wild facial faces are unstable and change for different photos while the last one is need of robustness and detailed 3D facial reconstruction. Different algorithms are described later which are used to reconstruct the 3D facial model from single photo using deep CNN and 3DMM and their comparison too. Pros and cons are explained briefly and then explained the selected different features for analyzing these algorithms. Finally, experimental setup and results of methods are described.

The four different algorithms that have been deployed for solving the above four challenges are as follows:

1. 3D dense face alignment (3DDFA).

2. Regressing robust and discriminative (RRD).

3. Volumetric regression network (VRN).

4. 3D detailed reconstruction (3DDR).

\section{METHODS}

This section describes the different methods which are designed for 3D facial reconstruction from an input single photo.

\subsection{D Dense Face Alignment (3DDFA) and Regressing Robust and Discriminative (RRD)}

3DDFA (Zhu, 2016) is used to fit the face model to the image via convolution neural network with project normalized coordinate code (PNCC). This algorithm is solved the first challenge that is listed above. An evaluation is conducted on 300W, AFLW and construction of AFLW200-3D database. This algorithm estimates the 3D facial reconstruction and facial alignment across large poses by using convolution neural network and landmarks. The input is the $100 \times 100 \times 3$ color image stacked by PNCC and the architecture consists of four convolution layers. Framework of face profiling is used to creating the large poses from medium poses to solve the second challenge.

When applied the single view 3D face reconstruction on wild facial faces, their 3D estimates are either unstable and change for different facial poses. So, Tran et al. (Tran, 2017) proposed to solve the problem described in third challenge. This algorithm has tested on LFW, YTF and IJB-A while best for face recognition in case of wild faces. This algorithm estimates the 3D facial shape and texture directly from an unconstrained 2D face image. For a given input image, it produces a standard .ply file of the face shape and texture. ResNet-101or Caffe deep network is used for regressing 3DMM shape and texture parameters under Linux.

\subsection{Volumetric Regression Network (VRN) and 3D Detailed Reconstruction (3DDR)}

Jackson et al. proposed method (Jackson, 2017) approaches the problem of reconstruction regarding segmentation. This algorithm specially estimates large pose 3D facial reconstruction using simple CNN architecture bypassing the training and testing of 3DMM. This method also performs direct regression of a volumetric representation of the 3D facial geometry from single input photo. The images are unconstrained and downloaded from websites that including arbitrary facial poses, expression and occlusion. Evaluation of this method is conducted on three different databases, namely AFLW2000-3D, BU-4DFE, and Florence. This method is developed under Linux and Torch 7 deep neural network framework is used. This architecture does not need accurate alignment but performs direct regression of a volumetric representation. 
Tran et al. proposed method (Tran, 2018) has ability to reconstruct the 3D facial model in detail under extreme conditions such as wearing glasses, hide by hairs etc. The algorithm is motivated by old age computer graphic concept named bump mapping (Blinn, 1978) that is used to separate the global shape from local details (wrinkles etc.). It naturally creates plausible details and better capability to produce the 3D facial with good texture model for out of plane rotations and occluded facial regions. The references set of this algorithm consists of $12 \mathrm{~K}$ unoccluded face images that are selected from the VGG and CASIA sets. The recent method of Sela et al. (Sela, 2017) close to this method that produces good detailed results when face become unoccluded but fails completely on occluded faces because of weak regularization. This algorithm estimate a coarse 3D face shape which acts as a foundation and then separately layer this foundation with details represented by a bump map. This method is developed under Linux and Pytorch deep neural network framework is utilized.

\section{DISCUSSION}

All the algorithms are state of the art on reconstruction of 3D face model by using deep neural network that are described above. We used Intel Core i7-7700K and NVIDIA GeForce GTX 1050 for the analysis of and generation of 3D facial model of different algorithms. 3DDFA method is the first algorithm which is used to reconstruct the 3D face model from single image using cascaded CNN. This algorithm has solved the problem of invisible landmarks in large poses and face alignment across large poses as well. The areas around mouth and nose are more sensitive while texture of 3D face shape is not so good. This method takes more than a minute to build one 3D solid facial model with pose and slightly texture to small extent.

The second algorithm is accurate, fast and robust that is used for estimating the parameters of 3DMM face shape from an input image using a deep CNN architecture. The areas around nose and mouth have very low errors and texture of 3D facial model is better than 3DDFA algorithm. This method is robust to recognize faces in the wild setting. This method takes less than 1 minute to build a 3D facial model with texture and slightly pose to some extent. It is failed in some cases when reconstructing the facial model such as beard hairs or facial hairs. This method is faster to predict the 3DMM parameters than 3DDFA.

The third approach works with unconstrained facial images of different poses, occlusions and facial expressions downloaded from the websites and reconstruct $3 \mathrm{D}$ face shape from single image. This CNN architecture for 3D segmentation is based on hourglass network (Newell, 2016). The volumetric architecture consists of two hourglasses modules.

Table 1. Comparison of Algorithms

\begin{tabular}{|l|c|c|c|c|}
\hline \multicolumn{1}{|c|}{ Algorithms } & 3DDFA & VRN & RRD & 3DDR \\
\hline $\begin{array}{l}\text { 3D Morphable Model } \\
\text { (3DMM) }\end{array}$ & Yes & Yes & Yes & Yes \\
\hline $\begin{array}{l}\text { Convolution neural Network } \\
\text { (CNN) Architecture }\end{array}$ & Yes & Yes & Yes & Yes \\
\hline Deep Neural Framework & No & Torch 7 & Caffe & Pytorch \\
\hline Operating System & Windows & Linux & Linux & Linux \\
\hline $\begin{array}{l}\text { Graphic Processing Unit } \\
\text { (GPU) }\end{array}$ & No & Yes & Yes & Yes \\
\hline Robustness & Low & Medium & Fast & Fast \\
\hline Complexity & Low & Medium & Medium & High \\
\hline Runtime & $75.7 m s$ & $69 m s$ & $88 \mathrm{~ms}$ & $50 \mathrm{~s}$ \\
\hline
\end{tabular}

The last method is complex than others but more efficient and robustness for $3 \mathrm{D}$ facial reconstruction from single photo by using deep neural frameworks with wild setting while using bump mapping concept and mid-level details. You can see the wrinkles and expression in every facial model as in the facial image while result seems good. It takes 50s per image producing 3D facial model and producing beautiful texture as compared to other methods. This algorithm is better for 3D printing faces, visual speech recognition and facial recognition, facial augmented reality and virtual reality. The comparison of different algorithms is shown in Table 1. 


\section{CONCLUSION}

The state of art methods regarding detailed 3D facial reconstruction from single image using deep neural network are explained. The recent approaches play an important in the field of computer vision community, graphics and deep learning field. Some algorithms produce better result across large poses, some better for robustness regarding texture parameter, some for occlusions and utmost conditions. Benefits and proposed solution of different problems is described. The performance of all the deep networks is used for different purposes such as facial expressions, occluded images and wild photos are discussed. Future work may include reviewing the 3D hair reconstruction from a single photo and developing a method and application as well for the reconstruction of 3D facial shape with hairs from single image by using deep neural networks.

\section{REFERENCES}

Blanz, V. and Vetter, T., 1999. Morphable model for the synthesis of 3D faces. In Proc. ACM SIGGRAPH Conf. Comput. Graphics, 1999.

Blinn, F. J., 1978. Simulation of wrinkled faces. Proc. ACM SIGGRAPH Conf. Comput. Graphics, 12(3):286-292, 1978.

Chinaev, N. et al, 2018. MobileFace: 3D face reconstruction with efficient CNN regression. arXiv preprint arXiv:1809.08809, 2013.

Feng, M. et al, 2018. 3D face reconstruction from light field images: A model free approach. In European Conf. Comput. Vision, 2018

Hassner, T. and Basri, R., 2006. Example based 3D reconstruction from single images. In Proc. Conf. Comput. Vision Pattern Recognition (CVPR) Workshops, IEEE, 2006.

Hassner, T. and Basri, R., 2013. Single view depth estimation from examples. arXiv preprint arXiv:1304.3915, 2013.

Jackson, A. S. et al, 2017. Large pose 3D face reconstruction from a single image via direct volumetric CNN Regression. Proc. Int. Conf. Comput. Vision, 2017.

Jiang, L. et al, 2018. 3D face reconstruction with geometry details from a single image. arXiv preprint arXiv:1702,05619, 2018.

Jourabloo, A. and Liu, X., 2016. Large-pose face alignment via cnn based dense 3D model fitting. In Proc. Conf. Comput. Vision Pattern Recognition (CVPR), 2016.

Kemelmacher-Shlizerman, I. and Basri, R., 2011. 3D face reconstruction from a single image using a single reference face shape. Trans. Pattern Anal. Mach. Intell., 33(2):394-405, 2011.

$\mathrm{Li}, \mathrm{C}$. et al, 2014. Intrinsic face image image decomposition with human face priors. In European Conf. Comput. Vision, pages 218-233. Springer, 2014.

Newell, A. et al, 2016. Stacked hourglass networks for human pose estimation. In European Conf. Comput. Vision, 2016.

Sela, M. et al, 2017. Unrestricted facial geometry reconstruction using image-to-image translation. In Proc. Int. Conf. Comput. Vision, 2017.

Tran, T. T. et al, 2017. Regressing robust and discriminative 3D morphable models with a very deep neural network. In Proc. Conf. Comput. Vision Pattern Recognition (CVPR), 2017.

Tran, T. T. et al, 2018. Extreme 3d face reconstruction: Seeing through occlusion. In Proc. Conf. Comput. Vision Pattern Recognition (CVPR), 2018.

Xing, Y. et al, 2018. A self-supervised bootstrap method for single-image 3D face reconstruction. arXiv preprint arXiv: 1812.05806, 2018.

Zhu, X. et al, 2016. Face alignment across large poses: A 3D solution. In Proc. Conf. Comput. Vision Pattern Recognition (CVPR), 2016. 